\title{
Reinhard B. Dettmeyer: Forensic Histopathology: Fundamentals and Perspectives
}

\author{
Springer, Heidelberg Dordrecht London New York, 2011, 478 pp. 370 figs. Hard cover. EUR \\ 139.95 (net price), CHF 201.00, USD 189.00, GBP 126.00 (ISBN: 978-3-642-20658-0)
}

\section{Heidi Pfeiffer}

Published online: 15 November 2011

(C) Springer-Verlag 2011

The present book constitutes a comprehensive update of $\mathrm{W}$. Janssen's work which came out a quarter of a century ago. Forensic histopathology plays an important role in forensic casework, so forensic pathologists have long been waiting for the publication of a work like this. In many cases, cause of death cannot be determined with certainty at autopsy without histological evidence. Moreover, histological evidence is of tremendous significance in answering other important questions in routine forensic work.

R.B. Dettmeyer is a specialist in both pathology and forensic medicine. He has several decades of experience in the field of histopathology. In this present work over the course of 20 chapters, he offers the reader profound insights into the areas of application of forensic histopathology as well as its significance. All major fields of routine forensic work are considered: histopathology of selected trauma, head, fire, electricity, lightning, radiation and gases, toxinand drug-induced pathologies including alcohol-related pathologies, selected medical malpractice cases, hypothermia, thrombosis and embolism, vitality and injury age, aspiration and inhalation, diagnosis of species, gender, age and identity, natural causes of death, forensic cytology and forensic neuropathology. In a further chapter, special staining and examination techniques are presented.

The 370 colour plates are marked by their high quality. The legends are informative and lucid. Each chapter is based on a synopsis of the latest literature on the subject.

This book is to be recommended to all physicians involved in forensic work as a new standard work on forensic histopathology.
H. Pfeiffer $(\square)$

Institute of Legal Medicine, University of Münster,

Roentgenstrasse 23,

48147 Münster, Germany

e-mail: Heidi.Pfeiffer@ukmuenster.de 